\title{
Diffuse Axonal Injury in Periventricular Leukomalacia as Determined by Apoptotic Marker Fractin
}

\author{
ROBIN L. HAYNES, SARAID S. BILLIARDS, NATALIA S. BORENSTEIN, JOSEPH J. VOLPE, AND HANNAH C. KINNEY
}

Departments of Pathology [R.L.H., S.S.B., N.S.B., H.C.K.], and Neurology [J.J.V.], Children's Hospital and Harvard Medical School, Boston, Massachusetts 02115

\begin{abstract}
Periventricular leukomalacia (PVL), the major substrate of neurologic deficits in premature infants, is associated with reduced white matter volume. Using immunomarkers of axonal pathology $[\beta$-amyloid precursor protein $(\beta$-APP) and apoptotic marker fractin], we tested the hypothesis that widespread (diffuse) axonal injury occurs in the gliotic white matter beyond the foci of necrosis in PVL, thus contributing to the white matter volume reduction. In a cohort of 17 control cases and 13 PVL cases with lesions of different chronological ages, diffuse axonal damage in PVL was detected by fractin in white matter sites surrounding and distant from acute and organizing foci of necrosis. Using $\beta$-APP, axonal spheroids were detected within necrotic foci in the acute and organizing (subacute) stages, a finding consistent with others. Interestingly, GAP-43 expression was also detected in spheroids in the necrotic foci, suggesting attempts at axonal regeneration. Thirty-one percent of the PVL cases had thalamic damage and $15 \%$ neuronal injury in the cerebral cortex overlying PVL. We conclude that diffuse axonal injury, as determined by apoptotic marker fractin, occurs in PVL and that its cause likely includes primary ischemia and trophic degeneration secondary to corticothalamic neuronal damage. (Pediatr Res 63: 656-661, 2008)
\end{abstract}

$\mathrm{T}^{\mathrm{s}}$ he principal pathology accounting for the predominance of neurodevelopmental disability in premature infants is periventricular leukomalacia (PVL) (1). This disability includes cognitive/behavioral deficits in 25-50\% and cerebral palsy in $5-10 \%$ of very low birth weight (VLBW) infants $(<1500 \mathrm{~g})$ (1). Neuroimaging studies of VLBW survivors suggest that the cerebral palsy is related to the focal necrotic lesions of PVL, whereas the cognitive/behavioral deficits correlate with more diffuse cerebral white matter injury $(2,3)$. PVL is defined as damaged immature cerebral white matter with periventricular focal necrosis ("focal" component) in association with diffuse reactive gliosis and microglial activation in the surrounding white matter ("diffuse" component) (4). A major cause of PVL is cerebral ischemia/reperfusion complicating respiratory compromise in the premature infant (1), potentially augmented by fetal infection/inflammation in at least some cases $(1,5,6)$. In long-term survivors with PVL, neuroimaging studies often demonstrate reduced cerebral white matter volume, impaired myelination, and ventriculomegaly $(7,8)$.

Received August 15, 2007; accepted January 4, 2008

Correspondence: Robin L. Haynes, Ph.D., Department of Pathology, Children's Hospital, Boston, MA 02115; e-mail: robin.haynes@childrens.harvard.edu

This work was supported by grants from the NINDS (PO1-NS38475) and NICHD (Children's Hospital Developmental Disabilities Research Center [P30-HD18655]).
The major focus of PVL research to date has been upon the role of premyelinating oligodendrocytes (preOLs) in its evolution, specifically testing the hypothesis that preOLs in the diffuse component are the key target of ischemic and/or inflammatory injury that results in myelin deficits $(9,10)$. It has long been recognized that axonal damage occurs within and at the periphery of the periventricular necrotic foci (the focal component) in PVL, and that this damage is characterized by spheroids immunopositive for $\beta$-amyloid precursor protein $(\beta$-APP) $(4,11-13)$. Yet, it is not clear if axonal injury occurs in the gliotic white matter surrounding or distant from the necrotic foci, that is, in the diffuse (nonnecrotic) component. Indeed, diffuse axonal injury could cause, at least in part, the reduced white matter volume seen in long-term survivors with PVL (14). In the following study, we tested the hypothesis that widespread and diffuse axonal injury occurs in the gliotic white matter that surrounds and/or extends beyond the characteristic foci of periventricular necrosis in PVL.

\section{MATERIALS AND METHODS}

Case selection. Cases were obtained from the autopsy service at Children's Hospital Boston and were classified as PVL based on the histopathologic criteria of damaged immature cerebral white matter with periventricular focal necrosis in association with diffuse reactive gliosis and microglial activation in the surrounding white matter (4). Control cases did not have PVL or other significant brain pathology upon standard histologic examination. Autopsy reports were reviewed for major clinical findings, systemic autopsy diagnoses, and neuropathologic findings. In addition, the brain microscopic sections were reviewed by the pediatric neuropathologist of the study (H.C.K.). PVL blocks (4\% paraformaldehyde-fixed) were selected for axonal analysis based on the presence in the block of one or more focally necrotic lesions. The stage of the necrosis was categorized by histopathologic criteria as acute, organizing, or chronic. Acute PVL was defined as focal periventricular necrosis characterized by coagulative necrosis (cytoplasmic hypereosinophilia and nuclear pyknosis of all tissue elements) and axonal spheroids; organizing PVL with focal periventricular necrosis characterized by macrophages; and chronic PVL characterized by focal cavitation or scar formation with axonal mineralization (4). Reactive astrocytes were noted in the surrounding white matter at all histopathologic stages. Large organizing cerebral cortical infarcts that involved the underlying white matter were included in the study as positive tissue controls for axonal damage, i.e., so-called "axonal cases." All brain tissues were obtained with parental permission for research according to the guidelines of the Human Protection Committee. This study was approved by the Institutional Review Board of Children's Hospital Boston.

Single-labeling immunocytochemistry in paraformaldehyde-fixed, paraffin-embedded tissue. Antibodies specific for the following markers were used in immunocytochemistry: $\beta$-APP (1:50; Chemicon, Temecula, CA); fractin

\footnotetext{
Abbreviations: $\boldsymbol{\beta}$-APP, $\beta$-amyloid precursor protein; GAP-43, growth-associated protein-43; pre-Ols, pre-myelinating oligodendrocytes; PVL, periventricular leukomalacia; VLBW, very low birth weight infants
} 
(1:500; BD Bioscience, San Jose, CA); and GAP-43 (1:100; Chemicon, Temecula, CA). Antigen retrieval for fractin and GAP-43 involved 10 min of microwave at $195^{\circ} \mathrm{F}$ in citrate buffer, $\mathrm{pH}$ 6.0. Antigen retrieval for $\beta$-APP involved heating sections in citrate buffer ( $\mathrm{pH}$ 6.0) to a boil, cooling for $5 \mathrm{~min}$, heating again to boil, and cooling for $20 \mathrm{~min}$. After retrieval, standard methods were applied (9). Negative controls omitted the primary antibodies. We qualitatively assessed immunostained sections with standard light microscopy. In representative cases, we mapped the distribution of axonal injury relative to the necrotic foci using the computer-based system Neurolucida (Microbrightfield Inc., Williston, VT).

\section{RESULTS}

Clinicopathologic information. We analyzed axonal pathology in the cerebral hemisphere of 13 cases of PVL compared with 17 control cases without PVL. Clinicopathologic information on all cases are listed in Table 1. Two cases were used as positive controls of known axonal injury: (1) a cerebral cortical infarct in an 8-month-old with a history of prematurity, surgical resection for necrotizing enterocolitis, and short gut syndrome; and (2) widespread acute neuronal necrosis of the cerebral cortex in a 12-month-old infant with foreign body aspiration and acute respiratory failure.

PVL cases analyzed with markers of axonal damage. Upon macroscopic examination, 7 of 13 (54\%) PVL cases had focal lesions in the periventricular regions, consisting of small cysts (up to $1 \mathrm{~cm}$ in diameter), dense scars, or "white spots" (i.e., focal necrosis with macrophagocytic infiltration). Upon microscopic examination, within the total 13 PVL cases studied, five cases had one or more acute focal periventricular lesions within a single tissue section or within different sections, four cases had one or more organizing foci, and four cases had chronic glial scars. Upon survey of all 13 PVL cases (15 lesions), axonal damage was detected in the focal component of PVL in 10 of 13 cases (11 of 15 lesions). Diffuse axonal damage was seen in 8 of the 13 cases, with variable degrees of severity (Table 2). One acute lesion (Case 1)

Table 1. Clinicopathologic information on PVL $(n=13)$ and control $(n=17)$ groups

\begin{tabular}{|c|c|c|}
\hline Clinicopathologic information & PVL & Control \\
\hline Mean postconceptional age (wks) & $43 \pm 16$ & $42 \pm 31$ \\
\hline Mean gestational age (wks) & $36 \pm 3$ & $32 \pm 7$ \\
\hline Mean postnatal age (wks) & $7.5 \pm 17$ & $10.5 \pm 27$ \\
\hline Postmortem interval range (hrs) (median) & $6-44(17)$ & $1.5-132(14)$ \\
\hline \multicolumn{3}{|l|}{ Cause of death $(n)$} \\
\hline Complications of prematurity & 4 & 6 \\
\hline Congenital diaphragmatic hernia & 1 & 1 \\
\hline Congenital heart disease & 2 & 1 \\
\hline Potter syndrome & 1 & 1 \\
\hline Idiopathic primary pulmonary hypertension & 1 & - \\
\hline Primary renal failure & 1 & - \\
\hline Inborn urea cycle defect & 1 & - \\
\hline Primary brainstem malformation & 1 & - \\
\hline $\begin{array}{l}\text { Marshall-Smith syndrome; acute respiratory } \\
\text { failure }\end{array}$ & 1 & - \\
\hline Noonan's syndrome & - & 1 \\
\hline Fetal hydrops & - & 1 \\
\hline Neonatal hepatic disease & - & 1 \\
\hline Immune thrombocytopenia & - & 1 \\
\hline Possible mitochondrial disorder & - & 1 \\
\hline Sudden unexplained death in childhood & - & 1 \\
\hline Trisomy 21 & - & 1 \\
\hline Unexplained stillbirth & - & 1 \\
\hline
\end{tabular}

Table 2. Summary of axonal findings in PVL cases with one of more focal necrotic lesions

\begin{tabular}{rllccccc}
\hline & & \multicolumn{3}{c}{ FOCAL } & & \multicolumn{2}{c}{ DIFFUSE } \\
\cline { 3 - 4 } \cline { 6 - 7 } Case & PVL & $\beta$-APP & Fractin & & $\beta$-APP & Fractin \\
\hline 1 & Acute & + & + & & + Moderate & - \\
1 & Acute & + & - & & - & - \\
2 & Acute & + & - & & - & + Rare \\
3 & Acute & NA & + & & NA & + Severe \\
4 & Acute & + & - & & - & + Severe \\
5 & Acute & + & - & & - & + Severe \\
6 & Organizing & + & - & & + & - \\
7 & Organizing & + & + & & + & + Severe \\
8 & Organizing & + & + & & - & + Severe \\
9 & Organizing & - & - & & - & - \\
9 & Organizing & - & - & & - & - \\
10 & Chronic & + Mineralized & + Mineralized & & - \\
11 & Chronic & - & - & & - & - \\
12 & Chronic & - & - & & - & - \\
13 & Chronic & + Mineralized & - & & - & - \\
\hline
\end{tabular}

Listed are the axonal findings for the 13 PVL cases arranged in chronological order of lesion type. Of those 13 PVL cases, 2 cases (Cases 1 and 9) had multiple lesions in different tissue blocks. The axonal findings described are associated with a particular lesion in an individual tissue block.

+ indicates the presence of a marker in axons; - , the lack of a marker in axons; NA, indicates that this information is not available.

showed focal axonal damage and no associated diffuse axonal damage. Within the PVL cases characterized as acute, $\beta$-APPimmunopositive spheroids were seen in five of six $(83 \%)$ acutely necrotic foci, whereas fractin-immunopositive spheroids were seen in only two of six foci (33\%) (Table 2).

Although $\beta$-APP-immunopositivity was rarely seen in the diffuse component of acute PVL cases, diffuse fractinimmunopositive axons were seen in the surrounding white matter in four of five cases (80\%) [four of six lesions (67\%)] (Table 2, Fig. 1). In three of these four cases, the axonal damage was widespread and severe extending out into the

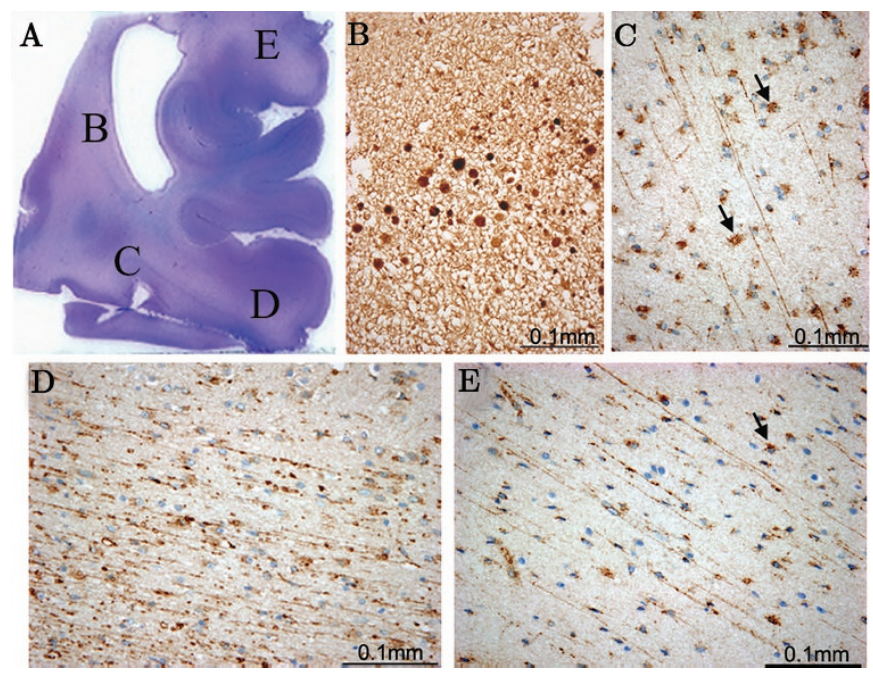

Figure 1. In an acute PVL case (44 PC weeks), a hemotoxylin and eosin stain $(\mathrm{H} \& \mathrm{E})(A)$ of a parieto-occipital white matter section indicates the presence of an acute necrotic lesion with $\beta$-APP expression in axonal spheroids $(B)$. In this same case, fractin positivity was seen in the diffuse gliotic component of PVL in the deep white matter $(C)$ as well as the intragyral white matter distant from the focal necrosis $(D, E)$. Also detected were fractin positive reactive astrocytes ( $C, E$ arrows). 


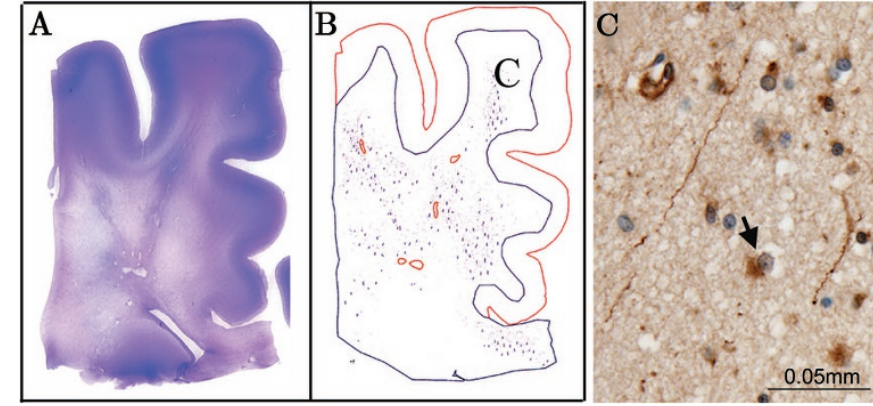

Figure 2. In an organizing PVL case (39 PC weeks), an H\&E stain of a posterior frontal section indicates the presence of multiple cysts within the deep white matter and widespread rarefaction of the diffuse gliotic component as seen by the pallor of the stain $(A)$. A Neurolucida image showing white matter with overlying cerebral cortex was drawn using this same section stained for fractin $(B)$. Red circles indicate focal microcysts while blue lines indicate fractin-positive axons. In this case, fractin positive axons can be detected throughout the white matter $(C)$, into the intragyral white matter and the corpus callosum. Fractinpositive astrocytes were detected within the diffuse component $(C$, arrow).

intragyral white matter and distant from the periventricular focus. In the majority of regions with central and intragyral axonal damage, the overlying cerebral cortex was histologically intact, without neuronal necrosis, obvious neuronal loss, and/or gliosis. In the cases with organizing PVL, three of four cases (75\%) [three of five lesions (60\%)] showed $\beta$-APPimmunopositive spheroids in the focal periventricular necrosis, whereas two of four cases $(50 \%)$ [two of five lesions (40\%)] showed fractin-immunopositivity. Within the diffuse component of the cases with organizing PVL, two of four $(50 \%)$ cases [two of five lesions (40\%)] showed diffuse $\beta$-APP-immunostaining, whereas two of four $(50 \%)$ cases [two of five lesions (40\%)] showed diffuse, severe fractinimmunostaining (Fig. 2). In the chronic PVL cases, $\beta$-APPand fractin-immunostaining was present in mineralized axons of the focal glial scars (Fig. 3) in two of four and one of four of the cases, respectively. No $\beta$-APP- or fractin-immunostaining was seen in the diffuse component in the cases with chronic PVL.

GAP-43 analysis in PVL. To examine potential axonal repair, we examined GAP-43 immunostaining in the focal and diffuse component of PVL in a subset of the PVL cases. In the focal component, GAP-43 was seen in axonal spheroids of six of seven acute lesions (86\%) (Fig. 4), and in one of five organizing focal lesions (20\%). No GAP-43 was seen in the
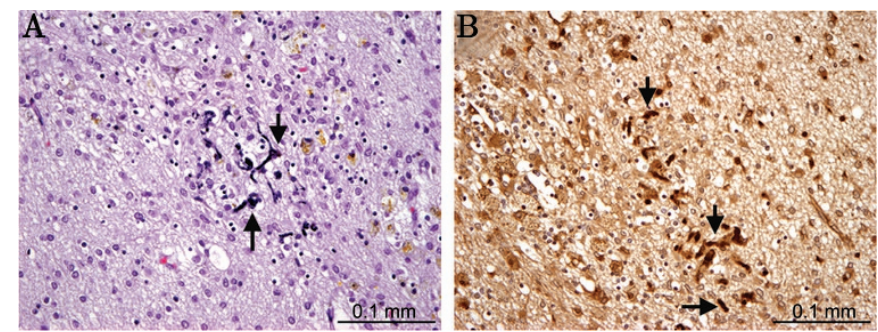

Figure 3. In a chronic PVL case (45 PC weeks), a glial scar contains mineralization of axonal elements ( $A$, arrow) as seen by $\mathrm{H} \& \mathrm{E}$, with fractin $(B$, arrow) expression strongly detectable within the mineralized axons.

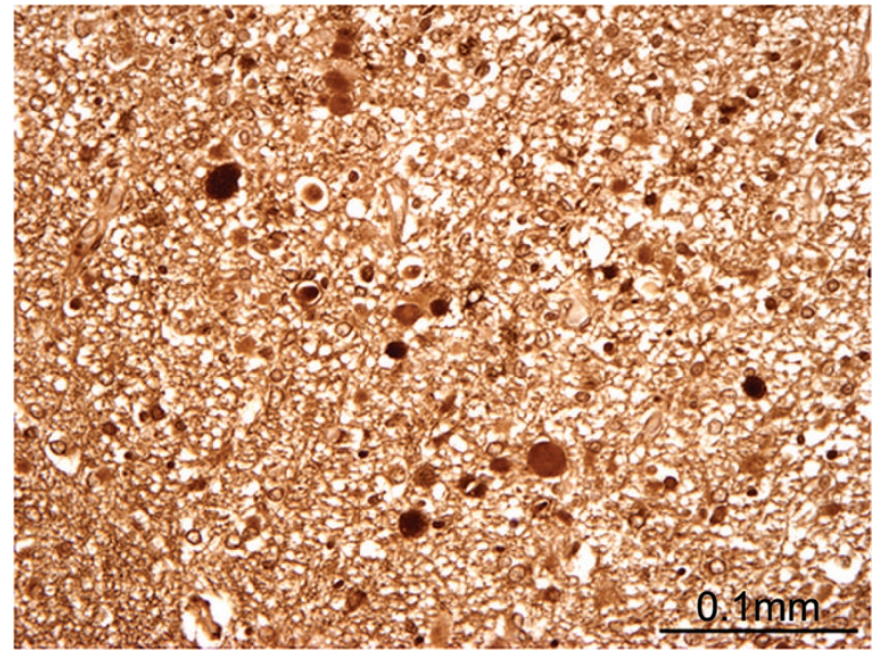

Figure 4. Expression of GAP-43 in axonal spheroids of acute necrotic foci in the white matter of a PVL case (44 PC weeks).

chronic PVL cases. We did not appreciate a change in GAP-43 immunostaining in the diffuse component compared with the central white matter of the control cases.

$P V L$ in association with thalamic and/or cortical damage. To assess the presence of associated gray matter injury in the cases with PVL, we reviewed the spectrum of findings of relevance to diffuse axonal injury in the cerebral white matter (Table 3). Approximately a third (31\%) of the PVL cases had thalamic gliosis, neuronal loss, and/or microinfarcts as determined by conventional histopathologic examination. Approximately a third $(31 \%)$ of PVL cases had cerebral cortical infarcts in the frontal, parietal, or occipital lobes. Of note, in the PVL cases associated with cerebral infarcts, the area of study for axonal damage in PVL was distant from the infarct, i.e., in a separate section with no overlying cortical damage. Visually appreciable neuronal loss was present in the overlying cerebral cortex in $15 \%$ of the PVL cases. None of the non-PVL, nonaxonal controls examined showed evidence of thalamic and/or cerebral cortical damage.

Axonal analysis in the control populations. In our analysis of control cases without PVL (or cerebral infarcts), we found no fractin- or $\beta$-APP-immunopositivity in the axons of the cerebral white matter, regardless of the age (Fig. 5). Examination of the "axonal" cases with cerebral infarcts showed fractin-immunopositivity in axons in the subcortical white matter, directly underlying the cortical damage (Fig. 6). APP positivity was also seen in each of the "axonal" cases but to a lesser degree than fractin (data not shown).

Table 3. Summary of thalamic and cortical pathology

\begin{tabular}{cccc}
\hline & $\begin{array}{c}\text { Thalamic } \\
\text { gliosis and/or } \\
\text { microinfarcts }\end{array}$ & $\begin{array}{l}\text { Cortical } \\
\text { infarcts }\end{array}$ & $\begin{array}{c}\text { Patchy cortical } \\
\text { neuronal loss* }\end{array}$ \\
\hline PVL & $4 / 13(31 \%)$ & $4 / 13(31 \%)$ & $2 / 13(15 \%)$ \\
Control & $0 / 18(0 \%)$ & $0 / 18(0 \%)$ & $0 / 18(0 \%)$ \\
\hline
\end{tabular}

$*$ The finding of patchy cortical neuronal loss refers to clusters of depopulation of three or more neurons, a semiqualitative observation. 


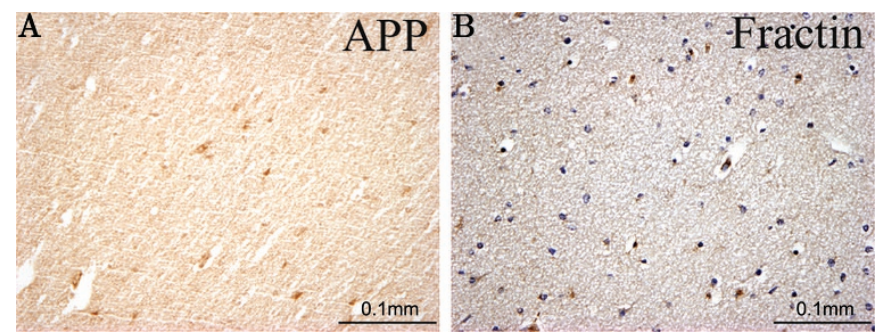

Figure 5. No detectable $\beta$-APP or fractin was seen in the white matter of control cases without PVL. Shown is a 37 PC week control case.

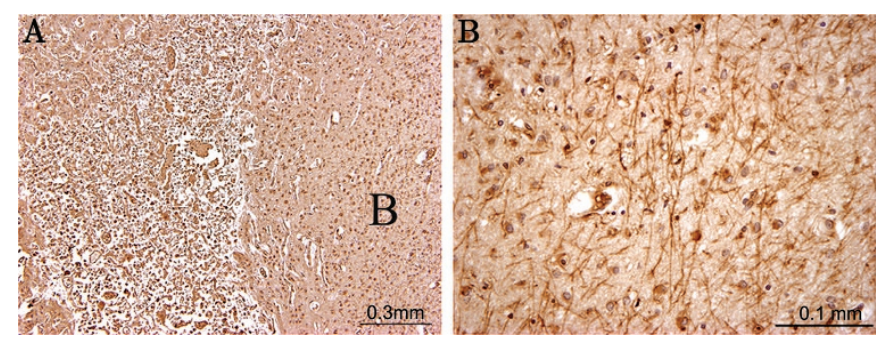

Figure 6. Fractin positivity in the white matter of an "axonal case" (40 PC weeks). (A) shows a low power of extensive cortical infarct with fractin positive axons detected at high power in the white matter underlying the cortical infarct $(B)$.

\section{DISCUSSION}

The major finding of this study is that diffuse axonal injury, as determined by the apoptotic marker fractin, occurs in the gliotic (non-necrotic) cerebral white matter in the acute and organizing stages of focal PVL. Axonal damage in PVL has long been recognized $(6,14)$, including the application of immunomarkers to $\beta$-APP $(11-13,15)$. The observations with fractin immunocytochemistry in this study provide new and distinct observations that further contribute to our knowledge of axonal damage in PVL. From our understanding of previous articles, $\beta$-APP positivity is reported within small and widespread foci of necrosis (i.e., coagulative or organizing necrosis), and at the peripheral edge of such necrotic foci (11-13). Using apoptotic marker fraction, we observed not only a different type of axonal injury (apoptosis-specific), but also a different site, i.e., not in focal or widespread foci of necrosis, or at the periphery of necrosis, but rather, in the non-necrotic white matter between necrotic foci, an area that, in our cases, showed no $\beta$-APP immunostaining. These sites are the so-called "diffuse" regions that are characterized by reactive astrocytes and activated microglia, but not macrophagocytic infiltration, rarefaction, vacuolation, or other features of widespread tissue dissolution and edema.

Axonal injury in PVL as determined by fractin and $\boldsymbol{\beta}$ - $\boldsymbol{A P P}$. In our survey of brain pathology in PVL, we found focal axonal damage in the necrotic component, seen primarily as $\beta$-APP-immunopositive spheroids. This finding is in agreement with others who have reported $\beta$-APP either in or on the periphery of the focal necrosis $(11-13,16)$. The key new finding in this study, however, is that, in addition to focal axonal damage, there is widespread axonal damage, far from the focal necrosis, in the diffusely, gliotic component of PVL, as demonstrated by the relatively new marker, fractin. Using fractin, we were able to detect severe axonal damage in regions where no $\beta$-APP-immunopositivity was apparent. Fractin, an antibody to the $32 \mathrm{kd}$ product of caspase-cleaved actin, is a marker of apoptosis-related events. It has been demonstrated in apoptotic cell bodies, in pontosubicular neuronal necrosis due to perinatal hypoxic-ischemic injury (17) and in degenerating cell bodies, axons, and dendrites in Alzheimer disease (18).

We found that axonal damage in the diffuse component of PVL correlates, to a degree, with the chronological age of the periventricular focal necrosis dated by histopathologic criteria (see above). This age-dependence likely reflects the timing of the expression of the markers, as well as the extensiveness of axonal damage and potential loss. The most extensive axonal fractin-immunopositivity occurred in the diffuse component of acute PVL cases. Given that acute focal lesions occur within $24-48 \mathrm{~h}$ of the insult (4), this finding is consistent with the expression of fractin in perinatal animal models of hypoxiaischemia, which peaks between 3 and $19 \mathrm{~h}$ after injury and falls to undetectable levels by $24-48 \mathrm{~h}$ (17). While we do see fractin-immunopositive axons in the diffuse component in two of five PVL lesions with organizing (subacute) focal necrosis, the lack of positivity in the remaining three potentially reflects a time-period beyond the expression of fractin, particularly given that the pathology of an organizing lesion extends for a week or more beyond the insult. Finally, in PVL cases with chronic foci with cavitation or scar formation, we saw no diffuse axonal damage by either fractin or $\beta$-APP immunostaining. Again, these latter lesions represent a "snapshot" of a time-period beyond the active expression of fractin and $\beta$-APP. Nevertheless, the lack of axonal damage seen at this "end-stage" could potentially reflect an irreversible loss of axons in the white matter. Other methods are needed to address the question of axonal loss.

In our study, we observed $\beta$-APP-immunostaining predominately in the foci of necrosis, whereas we see fractinimmunostaining predominately in the diffuse component. This difference likely reflects the nature of the marker and the regulation of its expression. $\beta$-APP, a constitutive component of neurons, accumulates upon injury to a level that is detectable by immunocytochemistry. In axonal transection, $\beta$-APP accumulates in the proximal axonal ends. Considering the nature of the necrotic lesion with destruction of all cellular elements, axons seem to be transected, with $\beta$-APP accumulation at the point of transection. Because fractin is a marker of apoptotic and not necrotic events, the appearance of fractin is seen distant from the focal lesion-in distal sites where apoptotic events are likely occurring. Indeed, these two markers suggest that there are at least two types of mechanisms of axonal injury: one involving focal periventricular necrosis in vulnerable distal end-fields of vascular perfusion, and another involving primary injury because of lesser degrees of ischemia/reperfusion and apoptosis to axons diffusely coursing through the white matter in the prenumbra. In regard to our finding of fractin immunopositivity in axons, rather than in the neuronal cell body, evidence of local activation of caspases in axons and neurites, independent of the cell body, has been shown in models of HIV-associated sensory neuropathy (19) and Alzheimer's disease (20). 
Axonal regeneration and/or degeneration in PVL. A question arises about the reversibility of diffuse axonal damage in PVL. Is a $\beta$-APP- or fractin-immunopositive axon destined to degenerate and die, or can the damage be reversed and the axon spared in the natural progression of the disease? As stated $\beta$-APP-immunopositive spheroids in the focal necrotic lesion likely reflect transected axons and irreversible axonal damage at the point of transection. The upregulation of GAP-43 in axonal spheroids in acute and organizing lesions, however, suggests an attempt at repair or regeneration by axons in the focal necrosis. A similar finding is found in traumatic brain injury in the adult cat (21) and experimental optic nerve injury (22).

Although little is reported on the specific reversibility of fractin as a marker, the reversibility of apoptosis-induced caspase activation has been reported (23) and is dependent on the expression of antiapoptotic proteins. Whether the presence of fractin in axons definitely "marks" a dying axon in PVL is unknown. However, in an animal model of axotomy of the medial forebrain bundle, fractin-immunopositive fibers double-labeled with fluoro-jade $\mathrm{B}$, a marker of neuronal degeneration (24), suggesting that fractin-immunostained fibers are undergoing irreversible degeneration. Of note, we also detected fractin-immunopositive astrocytes in the diffuse white matter of PVL. Although the significance of this is unknown, it does suggest that caspase-activation in astrocytes, which are known to be more resistant to apoptosis than neurons, may be reversible given the increased levels of antiapoptotic proteins expressed in astrocytes under ischemic conditions $(25,26)$.

Primary and secondary axonal injury in PVL. The cause of the diffuse axonal injury in PVL is likely complex with no one single mechanism. In PVL, the role of hypoxic-ischemic injury in the pathogenesis of the cerebral white matter damage is well-documented in animal models and human studies $(10,27)$. Direct, primary injury to the axon in the diffuse component of PVL is thus likely due to hypoxia-ischemia based on the recognized hypoxic-ischemic insults to developing axons in animal models $(28,29)$. The diffuse axonal damage may also reflect degeneration secondary to the death of neuronal cell bodies in the cerebral cortex and/or subcortical regions (thalamus/basal ganglia/brainstem) whose axons project to and from the cerebral cortex. This idea is supported by modern neuroimaging studies of term-equivalent infants, older infants, and children with PVL in which reduced volumes of the cerebral cortex, thalamus, and/or basal ganglia are found (see below), suggesting a loss of neurons and/or neuropil in these gray matter regions $(30,31)$. Up to one-third of the PVL cases in the present study demonstrated variable degrees of thalamic and/or cerebral cortical injury, thus suggesting that primary axonal damage in these cases was complicated by retrograde axonal degeneration because of neuronal cell injury or loss. PVL cases with cerebrocortical infarcts indicate that axonal injury in any one part of the brain may be due to different causes, and compound the burden of axonal injury, irrespective of the mechanism.

Diffuse axonal injury in the pathogenesis of myelin deficits in $\boldsymbol{P V L}$. The hypomyelination seen in long-term survivors of PVL has been thought to involve primary injury and/or loss of preOLs in the white matter, resulting in a subsequent dysfunction or loss of actively myelinating OLs (32). This hypothesis is supported by evidence of a qualitative preOL loss in PVL in a subset of cases $(9,33)$, but is not supported by evidence of OL preservation in a larger set of cases, as determined with OL cell density measurements using OLIG2, pan-marker of OL lineage (34). With OL loss or damage in PVL still a possibility, we support the hypothesis that the hypomyelination in PVL as well as the diffuse axonal damage seen by us, is secondary to this OL loss/damage (14), given the role for myelin-related proteins and OL-specific signals in long-term viability, thickness, and conduction of axons $(35,36)$. While axonal damage secondary to OL damage is one possibility in PVL, a second hypothesis involves primary injury to the axon resulting in a decrease in axonal signals to OLs necessary for proper and complete myelination (14).

Relation to results of advanced MRI studies of VLBW infants. Studies of premature infants by advanced MRI techniques reveal abnormalities consistent with axonal damage. Diffusion tensor imaging of VLBW infants, as early as term equivalent age $(37,38)$ and then later in childhood $(39,40)$ demonstrate diminished relative anisotropy, an MRI measure of preferred directionality of diffusion. This impairment in anisotropic diffusion could be caused by either a disturbance of axonal number, size, packing, or axonal membranes or intracellular constituents (41) or alternatively, a disturbance in axonal ensheathment by OLs. Definitive correlations between pathologic findings concerning axonal injury and the diffusion tensor MRI data cannot be made without MRI data obtained at or close to the time of autopsy. Although such information was not available in this study, our findings have implications for the understanding of the above advanced MRI studies.

In conclusion, further research is necessary to understand the pathogenesis of diffuse axonal damage in PVL, including whether or not this damage ultimately results in irreversible axonal loss and impaired neuronal function. Research is also needed in how to prevent axonal injury in PVL to improve the neurologic outcome of premature infants.

\section{REFERENCES}

1. Volpe JJ 2001 Neurobiology of periventricular leukomalacia in the premature infant. Pediatr Res 50:553-562

2. Dyet LE, Kennea N, Counsell SJ, Maalouf EF, Ajayi-Obe M, Duggan PJ, Harrison M, Allsop JM, Hajnal J, Herlihy AH, Edwards B, Laroche S, Cowan FM, Rutherford MA, Edwards AD 2006 Natural history of brain lesions in extremely preterm infants studied with serial magnetic resonance imaging from birth and neurodevelopmental assessment. Pediatrics 118:536-548

3. Woodward LJ, Anderson PJ, Austin NC, Howard K, Inder TE 2006 Neonatal MRI to predict neurodevelopmental outcomes in preterm infants. N Engl J Med 355:685694

4. Kinney HC, Armstrong DL 2002 Perinatal Neuropathology. In: Graham DI, Lantos PE (eds) Greenfield's Neuropathology. Arnold, London, pp 557-559

5. Rezaie P, Dean A 2002 Periventricular leukomalacia, inflammation and white matter lesions within the developing nervous system. Neuropathology 22:106-132

6. Kadhim H, Evrard P, Kahn A, Prez C, Bonnier C, Sebire G 2005 Insights into etiopathogenic mechanisms involved in perinatal cerebral injury: implications for neuroprotection. In: Fong H (ed) Focus on Cerebral Palsy Research. Nova Science Publishers, Inc, Hauppauge, NY, pp 1-26

7. Melhem ER, Hoon AH Jr, Ferrucci JT Jr, Quinn CB, Reinhardt EM, Demetrides SW, Freeman BM, Johnston MV 2000 Periventricular leukomalacia: relationship between lateral ventricular volume on brain MR images and severity of cognitive and motor impairment. Radiology 214:199-204

8. Skranes JS, Martinussen M, Smevik O, Myhr G, Indredavik M, Vik T, Brubakk AM 2005 Cerebral MRI findings in very-low-birth-weight and small-for-gestational-age children at 15 years of age. Pediatr Radiol 35:758-765 
9. Haynes RL, Folkerth RD, Keefe RJ, Sung I, Swzeda LI, Rosenberg PA, Volpe JJ, Kinney HC 2003 Nitrosative and oxidative injury to premyelinating oligodendrocytes in periventricular leukomalacia. J Neuropathol Exp Neurol 62:441-450

10. Back SA, Han BH, Luo NL, Chricton CA, Xanthoudakis S, Tam J, Arvin KL, Holtzman DM 2002 Selective vulnerability of late oligodendrocyte progenitors to hypoxia-ischemia. J Neurosci 22:455-463

11. Arai Y, Deguchi K, Mizuguchi M, Takashima S 1995 Expression of beta-amyloid precursor protein in axons of periventricular leukomalacia brains. Pediatr Neurol 13:161-163

12. Meng SZ, Arai Y, Deguchi K, Takashima S 1997 Early detection of axonal and neuronal lesions in prenatal-onset periventricular leukomalacia. Brain Dev 19:480_ 484

13. Deguchi K, Oguchi K, Takashima S 1997 Characteristic neuropathology of leukomalacia in extremely low birth weight infants. Pediatr Neurol 16:296-300

14. Dammann O, Hagberg H, Leviton A 2001 Is periventricular leukomalacia an axonopathy as well as an oligopathy? Pediatr Res 49:453-457

15. Hirayama A, Okoshi Y, Hachiya Y, Ozawa Y, Ito M, Kida Y, Imai Y, Kohsaka S, Takashima S 2001 Early immunohistochemical detection of axonal damage and glia activation in extremely immature brains with periventricular leukomalacia. Clin Neuropathol 20:87-91

16. Deguchi K, Oguchi K, Matsuura N, Armstrong DD, Takashima S 1999 Periventricular leukomalacia: relation to gestational age and axonal injury. Pediatr Neuro $20 \cdot 370-374$

17. Rossiter JP, Anderson LL, Yang F, Cole GM 2002 Caspase-3 activation and caspase-like proteolytic activity in human perinatal hypoxic-ischemic brain injury. Acta Neuropathol 103:66-73

18. Yang F, Sun X, Beech W, Teter B, Wu S, Sigel J, Vinters HV, Frautschy SA, Cole GM 1998 Antibody to caspase-cleaved actin detects apoptosis in differentiated neuroblastoma and plaque-associated neurons and microglia in Alzheimer's disease. Am J Pathol 152:379-389

19. Melli G, Keswani SC, Fischer A, Chen W, Hoke A 2006 Spatially distinct and functionally independent mechanisms of axonal degeneration in a model of HIV associated sensory neuropathy. Brain 129:1330-1338

20. Ivins KJ, Bui ET, Cotman CW 1998 Beta-amyloid induces local neurite degeneration in cultured hippocampal neurons: evidence for neuritic apoptosis. Neurobiol Dis $5: 365-378$

21. Christman CW, Salvant JB Jr, Walker SA, Povlishock JT 1997 Characterization of a prolonged regenerative attempt by diffusely injured axons following traumatic brain injury in adult cat: a light and electron microscopic immunocytochemical study. Acta Neuropathol 94:329-337

22. Yin Y, Henzl MT, Lorber B, Nakazawa T, Thomas TT, Jiang F, Langer R, Benowitz LI 2006 Oncomodulin is a macrophage-derived signal for axon regeneration in retinal ganglion cells. Nat Neurosci 9:843-852

23. Green DR, Beere HM 2001 Apoptosis. Mostly dead. Nature 412:133-135

24. El-Khodor BF, Burke RE 2002 Medial forebrain bundle axotomy during development induces apoptosis in dopamine neurons of the substantia nigra and activation of caspases in their degenerating axons. J Comp Neurol 452:65-79

25. Xu L, Sapolsky RM, Giffard RG 2001 Differential sensitivity of murine astrocytes and neurons from different brain regions to injury. Exp Neurol 169:416-424
26. Chen XQ, Fung YW, Yu AC 2005 Association of 14-3-3gamma and phosphorylated bad attenuates injury in ischemic astrocytes. J Cereb Blood Flow Metab 25:338-347

27. Greisen G, Borch K 2001 White matter injury in the preterm neonate: the role of perfusion. Dev Neurosci 23:209-212

28. Underhill SM, Goldberg MP 2007 Hypoxic injury of isolated axons is independen of ionotropic glutamate receptors. Neurobiol Dis 25:284-290

29. McCarran WJ, Goldberg MP 2007 White matter axon vulnerability to AMPA/ kainate receptor-mediated ischemic injury is developmentally regulated. J Neurosci 27:4220-4229

30. Ricci D, Anker S, Cowan F, Pane M, Gallini F, Luciano R, Donvito V, Baranello G, Cesarini L, Bianco F, Rutherford M, Romagnoli C, Atkinson J, Braddick O, Guzzetta F, Mercuri E 2006 Thalamic atrophy in infants with PVL and cerebral visual impairment. Early Hum Dev 82:591-595

31. Srinivasan L, Dutta R, Counsell SJ, Allsop JM, Boardman JP, Rutherford MA, Edwards AD 2007 Quantification of deep gray matter in preterm infants at term equivalent age using manual volumetry of 3-tesla magnetic resonance images. Pediatrics 119:759-765

32. Volpe JJ 2001 Neurology of the Newborn. WB Saunders Company, Philadelphia

33. Back SA, Luo NL, Mallinson RA, O’Malley JP, Wallen LD, Frei B, Morrow JD, Petito CK, Roberts CT Jr, Murdoch GH, Montine TJ 2005 Selective vulnerability of preterm white matter to oxidative damage defined by F2-isoprostanes. Ann Neurol 58:108-120

34. Billiards SS, Haynes RL, Folkerth RD, Borenstein NS, Trachtenberg FD, Rowitch DH, Ligon KL, Volpe JJ, Kinney HC 2008 Oligodendrocyte and myelin analysis in periventricular leukomalacia in the human premature infant. Brain Pathol, in press

35. Bjartmar C, Yin X, Trapp BD 1999 Axonal pathology in myelin disorders. J Neurocytol 28:383-395

36. Roy K, Murtie JC, El-Khodor BF, Edgar N, Sardi SP, Hooks BM, Benoit-Marand M, Chen C, Moore H, O'Donnell P, Brunner D, Corfas G 2007 Loss of erbB signaling in oligodendrocytes alters myelin and dopaminergic function, a potential mechanism for neuropsychiatric disorders. Proc Natl Acad Sci USA 104:8131-8136

37. Huppi PS, Murphy B, Maier SE, Zientara GP, Inder TE, Barnes PD, Kikinis R, Jolesz FA, Volpe JJ 2001 Microstructural brain development after perinatal cerebra white matter injury assessed by diffusion tensor magnetic resonance imaging. Pediatrics 107:455-460

38. Counsell SJ, Shen Y, Boardman JP, Larkman DJ, Kapellou O, Ward P, Allsop JM, Cowan FM, Hajnal JV, Edwards AD, Rutherford MA 2006 Axial and radial diffusivity in preterm infants who have diffuse white matter changes on magnetic resonance imaging at term-equivalent age. Pediatrics 117:376-386

39. Vangberg TR, Skranes J, Dale AM, Martinussen M, Brubakk AM, Haraldseth O 2006 Changes in white matter diffusion anisotropy in adolescents born prematurely. Neuroimage 32:1538-1548

40. Thomas B, Eyssen M, Peeters R, Molenaers G, Van Hecke P, De Cock P, Sunaert S 2005 Quantitative diffusion tensor imaging in cerebral palsy due to periventricular white matter injury. Brain 128:2562-2577

41. Prayer D, Barkovich AJ, Kirschner DA, Prayer LM, Roberts TP, Kucharczyk J, Moseley ME 2001 Visualization of nonstructural changes in early white matter development on diffusion-weighted MR images: evidence supporting premyelination anisotropy. AJNR Am J Neuroradiol 22:1572-1576 